\title{
BOUNDARY VALUE CONTROLLABILITY AND OBSERVABILITY PROBLEMS FOR THE WAVE AND HEAT EQUATION
}

\author{
K.-D. WERNER ${ }^{1}$
}

(Received 8 April 1986; revised 12 November 1986)

\begin{abstract}
In this paper, we study controllability and observability problems for the wave and heat equation in a spherical region in $\mathbf{R}^{n}$, where the control enters in the mixed boundary condition. In the main result, we show that all "finite energy" initial states (i.e. $\left.\left(w_{0}, v_{0}\right) \in H^{1}(\Omega) \times L^{2}(\Omega)\right)$ can be steered to zero at time $T$, using a control $f \in L^{2}(\partial \Omega \times[0, T])$, provided $T>2$. On this basis, we use the duality principle to investigate initial observability for the wave equation. Applying the Fourier transform technique, we obtain controllability and observability results for the heat equation.
\end{abstract}

\section{Introduction}

In this paper, we consider the controllability problem for the wave equation $w_{t t}-\Delta w=0, t \geq 0, x \in \Omega$, where $\Omega$ is a spherical region in $\mathbf{R}^{n}$. The control force enters in the boundary condition $\partial w / \partial \nu+\sigma w=f$, where $\sigma>0$ is fixed. In our main result we show that all initial states $\left(w_{0}, v_{0}\right) \in H^{1}(\Omega) \times L^{2}(\Omega)$ can be steered to the zero state at time $T>2$ using the control $f$. On this basis, we investigate the initial state observability problem for the wave equation. Furthermore, we study observability problems for the heat equation $w_{t}-\Delta w=0$, $t \geq 0, x \in \Omega$ with the mixed boundary condition.

In many physical processes, control is applied at the boundary of the spatial region, in which the process evolves. The wave equation plays an important role in the study of structural vibrations, tubular catalytic reactions, etc. Boundary

\footnotetext{
${ }^{1}$ Institute of Geometry and Practical Mathematics, Technical University of Aachen, 5100 Aachen, Federal Republic of Germany

(C) Copyright Australian Mathematical Society 1988, Serial-fee code 0334-2700/88
} 
controllability and (final state) observability of the parabolic equation $w_{t}-\Delta w=$ 0 has applications in heat transfer and diffusion processes.

Following the steps of [9], we can weaken the assumption $\left(w_{0}, v_{0}\right) \in H^{2}(\Omega) \times$ $H^{1}(\Omega)$, which was imposed on the initial conditions in [10, p. 81]. More precisely, we can show that finite energy initial states, i.e. $\left(w_{0}, v_{0}\right) \in H^{1}(\Omega) \times L^{2}(\Omega)$ can be steered to the zero state with a control $f \in L^{2}(\partial \Omega \times[0, T])$ appearing in the mixed boundary condition, provided $T>2$. However, we obtain this stronger result only for the case in which the region $\Omega$ is the interior of the unit sphere in $\mathbf{R}^{n}(n \geq 2)$. This is in contrast to the weaker controllability result obtained in [15], where the specific geometry of $\Omega$ does not need to be prescribed. Further results concerning boundary controllability for the wave equation subject to various boundary conditions in other regions can be found in [4], [16]. For the case involving only one space dimension, complete results on these topics are available. For details, see [17], [18], [22] and the references cited therein.

In the case of the heat equation $w_{t}-\Delta w=0$, our first aim is to investigate observability problems. On this basis, a duality theorem proved in [4] can then be used to obtain the controllability result, where the control $f$ again enters in the mixed boundary condition. In [2], [3], [14], [19], [21], results concerning various observability problems for the heat equation in one space dimension or higher dimensions are established. For more details concerning controllability results see [6], [7].

This paper is organized as follows. In Section 2, we describe the abstract linear systems and specify the boundary control problem for the wave equation. A brief summary of preparatory results is also given. The underlying results, which lead to the definition of certain weak solutions of the wave equation established by the technique of transposition, are described in detail. On this basis, we proceed in Section 3 with the proof of our main result via solving an equivalent moment problem. We shall, however, indicate only the important steps in the proof, because the procedure is similar to that given in [9]. With the aid of this main result, initial state observability for the wave equation is obtained in Section 4, applying a duality theorem. Finally, in Section 5 , we shall deal with the heat equation again subject to the mixed boundary condition. Hereby we use the fact that the boundary control problem of the heat equation is closely linked to the corresponding boundary control problem for the wave equation.

\section{Preparatory results}

Let $X, Y, Z$ be Banach spaces. Consider the abstract linear system

$$
\begin{aligned}
& X \supset D(C) \stackrel{C}{\longrightarrow} Y \\
& F \downarrow \\
& \text { Z }
\end{aligned}
$$


where the 'observation' operator $C: X \rightarrow Y$ is linear with dense domain $D(C)$ and $F$ is linear and bounded. The system

$$
X \supset D(C) \stackrel{C}{\rightarrow} Y
$$

is a specialisation of (2.1) obtained by taking $F$ to be the identity operator on $X$. Together with (2.1) and (2.2) we consider the dual systems

$$
\begin{aligned}
& F^{*} \uparrow \\
& \stackrel{Z^{*}}{Z^{*}}
\end{aligned}
$$

or when $F$ is the identity on $X$

$$
X^{*} \stackrel{C^{*}}{\longleftarrow} D\left(C^{*}\right) \subset Y^{*},
$$

obtained by letting $X^{*}, Y^{*}$ and $Z^{*}$ denote the conjugate spaces of $X, Y$ and $Z$ respectively. $C^{*}$ and $F^{*}$ are taken to be the adjoint operators for $C$ and $F$.

With this notation, we give a general definition of observability and controllability problems.

DEFINITION 2.1. The system (2.1) is (continuously) $F$-observable if there is a constant $K \geq 0$ such that

$$
\|F x\|_{Z} \leq K\|C x\|_{Y}, \quad x \in D(C) .
$$

The system (2.2) is observable (i.e. initial state observable), if

$$
\|x\|_{X} \leq K\|C x\|_{Y}, \quad x \in D(C) .
$$

DEFINITION 2.2. Let $R(A)$ denote the range of the operator $A$. Then system (2.3) is $F^{*}$-controllable, if

$$
R\left(F^{*}\right) \subseteq R\left(C^{*}\right)
$$

and the system (2.4) is (exactly) controllable, if

$$
X^{*} \subseteq R\left(C^{*}\right)
$$

We now state a theorem relating observability and controllability.

THEOREM 2.3. (a) System (2.1) is F-observable if and only if system (2.3) is $F^{*}$-controllable.

(b) System (2.2) is observable if and only if system (2.4) is (exactly) controllable.

PROOF. For part (a) see [4, Theorem 2.3] and for (b) see [4, Theorem 2.1].

We now pose the boundary control problem for the wave equation. Consider the initial boundary value problem consisting of the equation

$$
\frac{\partial^{2} w}{\partial t^{2}}-\sum_{k=1}^{n} \frac{\partial^{2} w}{\partial x_{K}^{2}}=0, \quad x \in \Omega, t \geq 0
$$


where $\Omega=\left\{x \in \mathbf{R}^{n},\|x\|_{e}<1\right\}$ and $\partial \Omega=\Gamma=\left\{x \in \mathbf{R}^{n},\|x\|_{e}=1\right\},\|\cdot\|_{e}$ denotes the Euclidean norm.

The boundary condition is given by

$$
\frac{\partial w}{\partial \nu}(x, t)+\sigma w(x, t)=f(x, t), \quad x \in \Gamma, t \geq 0, \sigma>0 \text { fixed }
$$

where $\nu$ denotes the unit outward normal vector to $\Gamma$ and

$$
f \in L^{2}(\Gamma \times[0, T])
$$

for some $T>0$. The initial conditions are

$$
w(x, 0)=w_{0}(x) \in H^{1}(\Omega), \quad \frac{\partial w}{\partial t}(x, 0)=v_{0}(x) \in L^{2}(\Omega),
$$

where $H^{1}(\Omega)$ denotes the Sobolev space of order 1 . The Sobolev spaces $H^{r}(\Omega)$, $H^{s}(\Gamma)$ of real orders $r, s$ are defined as in [11].

Our control problem is defined as follows. Let $T$ be prescribed and $\left(w_{0}, v_{0}\right)$ be an initial state as specified in (2.8). Then, find a control $f$ satisfying (2.7) so that the solution of (2.5), (2.6) and (2.8) also satisfies

$$
w(x, T) \equiv w_{T}(x) \in H^{1}(\Omega), \quad \frac{\partial w}{\partial t}(x, T) \equiv v_{T}(x) \in L^{2}(\Omega) .
$$

Because of the time reversibility of (2.5), there is no loss of generality in assuming that

$$
w_{T}=v_{T}=0 .
$$

The main result of this paper is now summarized in

THEOREM 2.4. If $T>2$, the control problem is solvable with a control $f \in L^{2}(\Gamma \times[0, T])$ such that

$$
\|f\|_{\left.L^{2}(\Gamma \otimes \mid 0, T]\right)}^{2} \leq K\left(\left\|w_{0}\right\|_{H^{1}(\Omega)}^{2}+\left\|v_{0}\right\|_{L^{2}(\Omega)}^{2}\right),
$$

where $K$ is a positive constant independent of $w_{0}$ and $v_{0}$. If $T<2$, the control problem is not solvable in general.

The proof of this theorem is given in Section 3 .

Let the space $V$ defined by

$$
V=\left\{w \in H^{2}(\Omega): \partial w / \partial \nu+\left.\sigma w\right|_{\Gamma}=0, \sigma>0 \text { fixed }\right\} .
$$

First, we note from the trace theorem [11, Chap. 1, §3.3] that $w, \partial w / \partial \nu$ are defined on $\Gamma$ and are elements of the interpolation space $H^{3 / 2}(\Gamma), H^{1 / 2}(\Gamma)$ respectively. Furthermore, the map

$$
w \rightarrow(w|\Gamma, \partial w / \partial \nu| \Gamma)
$$


is a linear, continuous and surjective map of $H^{2}(\Omega)$ onto $H^{3 / 2}(\Gamma) \times H^{1 / 2}(\Gamma)$. Thus, $V$ is a closed subspace of $H^{2}(\Omega)$ and hence a Hilbert space with the inner product from $H^{2}(\Omega)$.

Next, we show that $V$ is dense in $H^{1}(\Omega)$, which is needed for Theorem 2.5. Let $f \in H^{1}(\Omega)$. It is sufficient to show that $f \perp V$ implies $f=0$. Since the Laplace operator $-\Delta$ is coercive on $H^{1}(\Omega)$, one can equip $H^{1}(\Omega)$ with an equivalent scalar product defined by

$$
(u, f)_{1} \equiv \int_{\Omega} \nabla u \nabla f d x+K_{0} \int_{\Omega} u f d x+\int_{\Gamma} \sigma u f d s,
$$

where $K_{0}$ denotes the coercivity constant. Let $u \in V, f \perp V$. Application of Green's formula yields

$$
\begin{aligned}
0=(u, f)_{1} & =\int_{\Omega} f\left(-\Delta+K_{0}\right) u d x+\int_{\Gamma}(\sigma u+\partial u / \partial \nu) f d s \\
& =\int_{\Omega} f\left(-\Delta+K_{0}\right) u d x, \quad \text { since } u \in V .
\end{aligned}
$$

In $[23$, p. 339$]$ it is shown that the boundary value problem

$$
-\Delta u+K_{0} u=g, \quad \sigma u+\partial u /\left.\partial \nu\right|_{\Gamma}=0, \quad \sigma>0 \text { fixed, }
$$

has a unique solution $u \in H^{2}(\Omega)$ for every $g \in L^{2}(\Omega)$. Hence,

$$
0=\left(u, f_{1}\right)=\int_{\Omega} f\left(-\Delta+K_{0}\right) u d x=\int_{\Omega} g f d x, \quad \forall g \in L^{2}(\Omega) .
$$

Thus, $f=0$, and the claim is proved.

In order to define a weak solution of $(2.5),(2.6),(2.8)$ we need the following theorem, whose proof can be found in [11, Chap. IV].

THEOREM 2.5. Let $T_{1}>0$ and suppose $\varphi_{0} \in V, \varphi_{1} \in H^{1}(\Omega), h \in$ $L^{2}\left(\left[0, T_{1}\right], H^{1}(\Omega)\right)$. Then there exists a unique function $\varphi$ such that

$$
\varphi(\cdot, t) \text { is continuous from }\left[0, T_{1}\right] \text { into } V \text { and } \varphi(\cdot, 0)=\varphi_{0},
$$

$\partial f / \partial t(\cdot, t)$ is continuous from $\left[0, T_{1}\right]$ into $H^{1}(\Omega)$ and $\partial \varphi / \partial t(\cdot, 0)=\varphi_{1}$, $\partial^{2} \varphi / \partial t^{2}$ and $\Delta \varphi$ lie in $L^{2}\left(0, T_{1} ; V^{\prime}\right)$ and satisfy in $L^{2}\left(0, T_{1} ; V^{\prime}\right)$

$$
\partial^{2} \varphi / \partial t^{2}-\Delta \varphi=h
$$

For the following it is important that one can identify the dual $V^{\prime}$ of $V$ (with respect to $H^{1}(\Omega)$ ) with $L^{2}(\Omega)$. In order to show this, we need the next two lemmas.

As usual, by a region in $\mathbf{R}^{n}$, we mean an open connected (nonempty) bounded set of points. 
LEMMA 2.6. Let $\Omega$ be a region in $\mathbf{R}^{n}$ and suppose that $\partial \Omega=\Gamma \in C^{2}$. Then there is a constant $C>0$ such that any function $f \in V$ satisfies the inequality

$$
\|f\|_{H^{2}(\Omega)} \leq C\|\Delta f\|_{L^{2}(\Omega)}
$$

Proof. Suppose that $f \in V$ and let $F$ denote $\Delta f$. Then $f(x)$ satisfies Poisson's equation

$$
\Delta f=F
$$

almost everywhere in $\Omega$. Moreover, by the definition of $V, \partial f / \partial \nu+\left.\sigma f\right|_{\partial \Omega}=0$. Multiplying $\Delta f=F$ by an arbitrary function $v \in H^{1}(\Omega)$ and applying Green's formula, we obtain

$$
\int_{\Omega} v \Delta f d x=\int_{\Omega} v F d x=-\left(\int_{\Omega} \nabla v \nabla f d x+\int_{\Gamma} \sigma v f d s\right) .
$$

Hence, $f$ is a generalized solution (cf. [13, p. 193]) of the mixed boundary value problem for equation (2.14). The inequality (2.13) follows then from Theorem 4 of $[13$, p. 217] (see footnote on that page).

LEMMA 2.7. Let $\Omega \subseteq \mathbf{R}^{n}$ be a bounded region of class $C^{1}$ and $\Gamma$ be the boundary with positive $(n-1)$-dimensional Lebesgue measure. Let $\sigma(s)$, defined on $\Gamma$, be a measurable bounded function with $\sigma(s)>0$. Then, the norms $\|f\|_{H^{1}(\Omega)}$ and

$$
|f|_{1} \equiv\left(\int_{\Omega} \sum_{k=1}^{n}\left|\partial f / \partial x_{k}\right|^{2} d x\right)^{1 / 2}+\left(\int_{\Gamma} \sigma(s)|f(x)|^{2} d s\right)^{1 / 2}
$$

are equivalent.

Proof. Theorem 28.5 of [20].

Let $H^{1}(\Omega)$ be equipped with the scalar product

$$
(f, g)_{1}=\int_{\Omega} \nabla f \nabla g d x+\int_{\Gamma} \sigma f g d s .
$$

The dual $V^{\prime}$ of $V$ with respect to $H^{1}(\Omega)$ in the norm $|\cdot|_{1}$ is defined as follows. Let $w \in H^{1}(\Omega)$ and define a continuous linear functional on $H^{1}(\Omega)$ by

$$
l_{w}(u)=(u, w)_{1}, \quad u \in H^{1}(\Omega) .
$$

For $u \in V \subseteq H^{2}(\Omega)$ we have with a positive constant $c$

$$
\|u\|_{V}=\|u\|_{H^{2}(\Omega)} \geq c|u|_{1}
$$

and hence

$$
\left|l_{w}(u)\right| \leq(1 / c)\|u\|_{V}|w|_{1},
$$


showing that $l_{w}$ is also a continuous linear functional on $V$. Since $V$ is a Hilbert space, there exists a unique $v(w) \in V$ such that

$$
l_{w}(u)=(u, v(w))_{V}, \quad u \in V .
$$

We define

$$
\|w\|_{V^{\prime}}=\|v(w)\|_{V}
$$

and $V^{\prime}$ itself to be the completion of $H^{1}(\Omega)$ with respect to this norm. Now,

$$
\|w\|_{V^{\prime}}=\|v(w)\|_{V}=\sup _{\mathbf{0} \neq u \in V}\left|(u, v(w))_{V}\right| /\|u\|_{V}
$$

Since

$$
(u, v(w))_{V}=(u, w)_{1}=\int_{\Omega} \nabla u \cdot \nabla w d x+\int_{\Gamma} \sigma u w d s
$$

(by (2.15) and (2.16)) integration by parts together with $u \in V$ leads to

$$
(u, v(w))_{V}=-\int_{\Omega}(\Delta u) w d x
$$

Thus,

$$
\begin{aligned}
& \|w\|_{V^{\prime}}=\sup _{0 \neq u \in V}\left|\int_{\Omega}(\Delta u) w d x\right| /\|u\|_{V} \\
& \leq \sup _{0 \neq u \in V}\left[\int_{\Omega}|\Delta u|^{2} d x\right]^{1 / 2}\|w\|_{L^{2}(\Omega)} /\|u\|_{V} \\
& \leq \sup _{0 \neq u \in V} K\|u\|_{V}\|w\|_{L^{2}(\Omega)} /\|u\|_{V}=K\|w\|_{L^{2}(\Omega)}
\end{aligned}
$$

for some fixed positive constant $K$. By Lemma 2.6,

$$
-\Delta u=w
$$

has a unique solution $\hat{u} \in V$ with $\|\hat{u}\|_{V} \leq \hat{K}\|w\|_{L^{2}(\Omega)}$. Equation (2.17) with $u=\hat{u}$ gives

$$
\left|\int_{\Omega}(\Delta \hat{u}) w d x\right|=\|w\|_{L^{2}(\Omega)}^{2} \leq\|\hat{u}\|_{V}\|w\|_{V^{\prime}} \leq \hat{K}\|w\|_{L^{2}(\Omega)}\|w\|_{V^{\prime}},
$$

so that

$$
\|w\|_{L^{2}(\Omega)} \leq \hat{K}\|w\|_{V^{\prime}} .
$$

Therefore, the $L^{2}(\Omega)$ and $V^{\prime}$ norms of $w \in H^{1}(\Omega)$ are equivalent. Since $V^{\prime}$ is the completion of $H^{1}(\Omega)$ with respect to the $V^{\prime}$ norm, it is then also the completion of $H^{1}(\Omega)$ with respect to the $L^{2}(\Omega)$ norm, which is $L^{2}(\Omega)$. Topologically, $V^{\prime}$ and $L^{2}(\Omega)$ are the same space, and the norms are equivalent. This completes the proof. 
Using Theorem 2.5, together with the technique of transposition and Green's formula as done in $[11$, p. 319$]$ and in [1], we obtain

THEOREM 2.8. Let $\left(w_{0}, v_{0}\right) \in H^{1}(\Omega) \times L^{2}(\Omega)$ and $f \in L^{2}\left(\Gamma \times\left[0, T_{1}\right]\right)$. Then for each solution of (2.12), as described in Theorem 2.5, together with

$$
\phi\left(x, T_{1}\right)=\frac{\partial \phi}{\partial t}\left(x, T_{1}\right)=0
$$

there exists a unique function $w \in L^{2}\left(\Omega \times\left[0, T_{1}\right]\right)$ such that the following relation is valid:

$$
\begin{aligned}
\int_{\Omega \times\left[0, T_{1}\right]} w\left(\frac{\partial^{2} \phi}{\partial t^{2}}-\Delta_{n} \phi\right) d x d t= & \int_{\Omega \times\left[0, T_{1}\right]} w h d x d t \\
= & \int_{\Omega}\left(v_{0}(x) \phi(x, 0)-w_{0}(x) \frac{\partial \phi}{\partial t}(x, 0)\right) d x \\
& +\int_{\Gamma \times\left[0, T_{1}\right]} f(x, t) \phi(x, t) d s d t
\end{aligned}
$$

We use (2.18) as the definition of weak solutions of (2.5).

REMARK 2.9. The identification of $V^{\prime}$ with $L^{2}(\Omega)$ together with (2.12) shows that the left-hand side of (2.18) is defined for $w \in L^{2}\left(\Omega \times\left[0, T_{1}\right]\right)$.

Because we can reverse the direction of time in the wave equation, the replacement of the initial conditions stated in Theorem 2.5 by the terminal conditions in Theorem 2.8 causes no problem. Since $w(\cdot, T)$ and $\partial w / \partial t(\cdot, T)$ are not very well defined by Theorem 2.8 , as required for our control problem, we replace condition (2.9) as follows. Let $T_{1}>T$, and extend $f$ from $[0, T]$ to $\left[0, T_{1}\right]$ by setting

$$
f(x, t) \equiv 0, \quad x \in \Gamma, t \in\left(T, T_{1}\right] .
$$

Then (2.9) is replaced by

$$
w(x, t) \equiv 0, \quad(x, t) \in \Omega \times\left[T, T_{1}\right] .
$$

Then (2.9) and (2.20) are equivalent for classical solutions of (2.5), (2.6), (2.8).

\section{Proof of Theorem 2.4}

Following the arguments of [9], we can transform the controllability problem (2.5), (2.6), (2.9) into a sequence of equivalent moment problems. For the sake of convenience, let us give a sketch of the procedure.

Denote by $\lambda_{K l}=\omega_{K l}^{2}, K=0,1, \ldots, l=1,2, \ldots$ the eigenvalues, and by $U_{K m l} \in L^{2}(\Omega)$ the orthonormalized eigenfunctions of

$$
\Delta U+\lambda U=0
$$


with the boundary condition

$$
\frac{\partial U}{\partial \nu}+\left.\sigma U\right|_{\Gamma}=0, \quad \sigma>0 \text { fixed. }
$$

Here $m, m=1,2, \ldots, h(K, p)=(2 K+p)(K+p-1) ! /(p ! K !)$, denotes the multiplicity of each eigenvalue. We remark that the eigenvalues are positive numbers. For each $K$, these numbers are defined by $\lambda_{K l}=\omega_{K l}^{2}$, where $\omega_{K l}$ satisfies

$$
(\sigma-p / 2) J_{K+p / 2}\left(\omega_{K l}\right)+\omega_{K l} J_{K+p / 2}^{\prime}\left(\omega_{K l}\right)=0, \quad l=1,2, \ldots, p=n-2 .
$$

Here $J_{\nu}$ is the Bessel function of the first kind with order $\nu$. Furthermore, $\omega_{K 1}<\omega_{K 2}<\cdots \rightarrow \infty$ and $\omega_{K_{1}} \rightarrow \infty$ as $K \rightarrow \infty$ for each $l=1,2, \ldots$ The corresponding normalized eigenfunctions are a triply indexed sequence given by

$$
\begin{aligned}
U_{K m l}(x) & =U_{K m l}(r, \Theta, \Phi)=R_{K l}(r) Y_{K m}(\Theta, \Phi), \\
& m=1,2, \ldots, h(K, p)=(2 K+p)(K+p-1) ! /(p ! K !) .
\end{aligned}
$$

Here $\Theta, \Phi$ are hyperspherical coordinates, $r \in[0,1]$ is the radial coordinate, $\Theta=\left\{\theta: 0 \leq \theta_{j} \leq \pi, j=1, \ldots, p\right\}$ is the coordinate of latitude and $\Phi \in[0,2 \pi)$ is the coordinate of longitude. The sequence $\left\{Y_{K m}(\Theta, \Phi)\right\}, m=1,2, \ldots, h(K, p)$ is then an orthonormal basis in $L^{2}(\Gamma)$ for the surface harmonics of degree $K$. The functions $R_{K l}(r)$ are defined by (see e.g. [5]):

$$
R_{K l}(r)=\frac{\sqrt{2} \omega_{K l} r^{-p / 2}}{\left\{\omega_{K l}^{2}-(K+\sigma)(K+p-\sigma)\right\}^{1 / 2}} \frac{J_{K+p / 2}\left(\omega_{K l} r\right)}{\left|J_{K+p / 2}\left(\omega_{K l}\right)\right|} .
$$

For each $K$, the functions are orthonormal (with weight function $r^{n-1}$ ) in $L^{2}(0,1)$. We now state a result which is needed for the proof of Theorem 2.4.

LEMMA 3.1. If $v_{0} \in H^{1}(\Omega)$, then the inequality

$$
\sum_{K, m, l} \lambda_{K l} v_{K m l}^{2}<\infty
$$

is satisfied, where

$$
v_{K m l}=\int_{\Omega} v_{0}(x) U_{K m l}(x) d x
$$

ProOF. Lemma 3.2 of [10].

Let $w$ be a solution of $(2.5),(2.6),(2.8)$ as defined in Theorem 2.8. The initial data have an expansion in $L^{2}(\Omega)$ :

$$
\left\{\begin{array}{l}
w_{0}(x) \\
v_{0}(x)
\end{array}\right\}=\sum_{K, m, l}\left\{\begin{array}{l}
w_{K m l} \\
v_{K m l}
\end{array}\right\} U_{K m l}(x)
$$

where

$$
\left\{\begin{array}{c}
w_{K m l} \\
v_{K m l}
\end{array}\right\}=\int_{\Omega}\left\{\begin{array}{c}
w_{0}(r, \Theta, \Phi) \\
v_{0}(r, \Theta, \Phi)
\end{array}\right\} U_{K m l}(r, \Theta, \Phi) r^{n-1} d r d s
$$


Let $T_{1}>T, g \equiv g(t) \in C^{\infty}[0, \infty)$ have support in $\left[T, T_{1}\right]$ (cf. Remark 2.9); define a function $g_{K m l} \in C^{\infty}(\Omega \times[0, \infty))$ by

$$
g_{K m l}(x, t) \equiv g(t) U_{K m l}(x) .
$$

Then, the solution of $\partial^{2} \phi / \partial t^{2}-\Delta_{n} \phi=g_{K m l}, \partial \phi / \partial \nu+\left.\sigma \phi\right|_{\Gamma}=0$, with zero terminal condition

$$
\phi\left(x, T_{1}\right)-\partial \phi / \partial t\left(x, T_{1}\right)=0
$$

is given by

$$
\begin{aligned}
& \phi(x, t)=\phi_{K m l}(x, t)=\left(\alpha_{1} \cos \left(\omega_{K l} t\right)+\alpha_{2}\left(1 / \omega_{K l}\right) \sin \left(\omega_{K l} t\right)\right) U_{K m l}(x) \\
& \alpha_{1}=\int_{T}^{T_{1}}\left(1 / \omega_{K l}\right) \sin \left(\omega_{K l} \tau\right) g(\tau) d \tau, \quad \alpha_{2}=-\int_{T}^{T_{1}} \cos \left(\omega_{K l} \tau\right) g(\tau) d \tau .
\end{aligned}
$$

Hence, $\phi \in C^{\infty}\left(\Omega \times\left[0, T_{1}\right]\right)$ satisfies the hypotheses of Theorem 2.8. Thus the solution $w(x, t)$ must satisfy (2.18) for such a $\phi(x, t)$. Now, by substituting $\phi(x, t)$ and $\partial \phi / \partial t(x, t)$ together with the expansions of $w_{0}, v_{0}$ into (2.18), we obtain

$$
\begin{gathered}
\alpha_{1}\left(\int_{\Gamma \times[0, T]} f(x, t) \cos \left(\omega_{K l} t\right) U_{K m l}(x) d s d t+v_{K m l}\right) \\
+\alpha_{2}\left(\int_{\Gamma \times[0, T]} f(x, t)\left(1 / \omega_{K l}\right) \sin \left(\omega_{K l} t\right) U_{K m l}(x) d s d t-w_{K m l}\right) \\
\quad=\int_{\Omega \times\left[T, T_{1}\right]} w(x, t) g_{K m l}(x, t) d x d t .
\end{gathered}
$$

If we expand $f(x, t)$ in terms of hyperspherical harmonics:

$$
f(x, t)=\sum_{K=0}^{\infty} \sum_{m=1}^{h(K, p)} f_{K m}(t) Y_{K m}(\Theta, \Phi), \quad t \in[0, T], x=(1, \Theta, \Phi) \in \Gamma,
$$

and use the orthonormality property of the sequence $\left\{Y_{K m}\right\}$ in $L^{2}(\Gamma)$, it is clear that the right hand side of (3.4) is zero, and hence (2.20) is satisfied if and only if the function $f_{K m} \in L^{2}[0, T]$ solves the infinite collection of moment problems:

$$
\begin{gathered}
\int_{0}^{T} f_{K m}(t) \exp \left(i \omega_{K l} t\right) d t=\left(-v_{K m l}+i \omega_{K l} w_{K m l}\right) / R_{K l}(1) \\
\int_{0}^{T} f_{K m}(t) \exp \left(-i \omega_{K l} t\right) d t=\left(-v_{K m l}-i \omega_{K l} w_{K m l}\right) / R_{K l}(1), \\
K=0,1, \ldots, l=1,2, \ldots, m=1,2, \ldots, h(k, p) .
\end{gathered}
$$

The solvability of such a moment problem depends on the value of $T$ and the properties of the nonnegative numbers $\omega_{K l}$.

The proof of the following lemma can be found in [8]. 
LEMMA 3.2. For fixed $p \geq 2, \sigma>0$, and $K=0,1, \ldots$, it is true that:

$$
\begin{array}{r}
\omega_{K, l+1}-\omega_{K, l}>\pi \\
\lim _{l \rightarrow \infty}\left(\omega_{K, l+1}-\omega_{K, l}\right)=\pi .
\end{array}
$$

This lemma implies that for each $K=0,1, \ldots$, the sequence $\left\{\omega_{K l}\right\}$ has an asymptotic gap $\pi$ and density

$$
D \equiv \lim _{l \rightarrow \infty}\left(l / \omega_{K l}\right)=1 / \pi .
$$

In $[12$, p. 3$]$ it is shown that the functions $\exp \left(i \omega_{K l} t\right), \exp \left(-i \omega_{K l} t\right), l=$ $1,2, \ldots$, are linearly dependent in $L^{2}[0, T]$ if $T<2 \pi D$. Thus, for

$$
S \equiv\left\{\exp \left(i \omega_{K l} t\right), \exp \left(-i \omega_{K l} t\right), i=1,2, \ldots\right\}
$$

there does not exist a biorthogonal set in $L^{2}[0, T]$ if $T<2 \pi D$. Since $D=1 / \pi$ in our case, this implies the statement of

LEMMA 3.3. If $T<2$, the moment problem (3.6), (3.7) has in general no solution.

Using Lemmas 6.3 and 6.4 of [9], we can prove the following theorem.

THEOREM 3.4. If $T>2$ and

$$
\begin{gathered}
\sum_{K, m, l}\left|v_{K m l}\right|^{2} \equiv a^{2}<\infty, \\
\sum_{K, m, l}\left|\omega_{K l} w_{K m l}\right|^{2} \equiv b^{2}<\infty
\end{gathered}
$$

then the moment problem has a solution $f(x, t) \in L^{2}(\Gamma \times[0, T])$ which satisfies

$$
\|f\|_{L^{2}(\Gamma \times[0, T])}^{2} \leq K_{1}\left(a^{2}+b^{2}\right),
$$

where the positive constant $K_{1}$ is independent of the coefficients $v_{K m l}, w_{K m l}$.

ProOF. Let the functions $f_{K m} \in L^{2}[0, T]$ be the solutions of the moment problems (3.6), (3.7) which exist for $T>2$ by Lemmas 6.3 and 6.4 of [9]. For each $K, m$ it follows from (3.6), (3.7) and Lemma 6.3 [9] that

$$
\left\|f_{K m}\right\|_{L^{2}[0, T]}^{2} \leq 2 \tilde{K}_{1} \sum_{l=1}^{\infty}\left(1 /\left|R_{K l}(1)\right|^{2}\right)\left(\left|v_{K m l}\right|^{2}+\left|\omega_{K l} w_{K m l}\right|^{2}\right),
$$

where the constant $\tilde{K}_{1}$ is determined by the positive number $T-2$ as well as the uniform lower bound $\pi$ of the asymptotic gap $\omega_{K, l+1}-\omega_{K, l}$. Recalling 
the definitions of $f(x, t)$ and $U_{K m l}(x)$, one sees that the orthonormality of the hyperspherical harmonics $Y_{K m}(\Theta, \Phi)$ implies that

$$
\begin{aligned}
& \left\|\sum_{K=0}^{\infty} \sum_{m=1}^{h(k, p)} f_{K m}(t) Y_{K m}(\Theta, \Phi)\right\|_{L^{2}(\Gamma \times[0, T])}^{2} \\
& \quad=\sum_{K=0}^{\infty} \sum_{m=1}^{h(K, p)}\left\|f_{K m}(t)\right\|_{L^{2}[0, T]}^{2} \\
& \quad \leq 2 \tilde{K}_{1} \sum_{K=0}^{\infty} \sum_{m=1}^{k(K, p)} \sum_{l=1}^{\infty}\left(1 /\left|R_{K l}(1)\right|^{2}\right)\left(\left|v_{K m l}\right|^{2}+\left|\omega_{K l} w_{K m l}\right|^{2}\right) \\
& \quad \equiv \sum_{K, m, l} a_{K m l}^{2} .
\end{aligned}
$$

Now, we need to show that

$$
\sum_{K, m, l} a_{K m l}^{2} \leq K_{1} \sum_{K, m, l}\left(\left|v_{K m l}\right|^{2}+\left|\omega_{K l} w_{K m l}\right|^{2}\right) \equiv K_{1}\left(a^{2}+b^{2}\right) .
$$

Thus, it is sufficient to show that there exists a positive number $K_{1}$ such that, for all $K, l$,

$$
2 \tilde{K}_{1}\left|R_{K l}(1)\right|^{-2} \leq K_{1} .
$$

For this, we recall that $\left|R_{K l}(1)\right|^{2}=2 \omega_{K l}^{2}\left\{\omega_{K l}^{2}-(K+\sigma)(K+p-\sigma)\right\}^{-1}$. Thus,

$$
\left|R_{K l}(1)\right|^{2} \geq 2\left(1+\sigma^{2} / \omega_{K l}^{2}\right)^{-1} \geq 2\left(1+\sigma^{2} / \omega_{K l}^{2}\right)^{-1},
$$

since $\omega_{K 1}<\omega_{K 2}<\cdots \rightarrow \infty$ (see [8, Theorem 1.1]). Using the fact that $\omega_{K l} \rightarrow \infty$ as $K \rightarrow \infty$ for $l=1,2, \ldots$ and $\omega_{K l}>0$ for all values $K, l$, we conclude that there exists a constant $\mu$ such that

$$
0<\mu \equiv \min \left\{\omega_{K l}, K=0,1, \ldots\right\} \text {. }
$$

Therefore,

$$
\left|R_{K l}(1)\right|^{2} \geq 2\left(1+\frac{\sigma^{2}}{\mu^{2}}\right)^{-1} \equiv c,
$$

which proves the inequality (3.11). Thus, the series (3.5) converges in $L^{2}(\Gamma \times[0, T])$ and represents a solution of the moment problem (3.6), (3.7). This completes the proof.

We are now in a position to prove our main theorem.

PROOF OF THEOREM 2.4. Lemma 3.1 shows that if $w_{0} \in H^{1}(\Omega)$, then the inequality (3.9) is satisfied. Of course, condition (3.8) indicates that $v_{0} \in L^{2}(\Omega)$. Theorem 3.4 now shows that there exists an $f \in L^{2}(\Gamma \times[0, T])$ which solves the moment problem (3.6), (3.7) provided $T>2$. In the derivation of the moment problem we see that this is equivalent to the controllability condition. 
The proof is complete.

The next lemma indicates that one can not, in general, obtain more information from Theorem 2.4 by using inequalities (3.8) and (3.9).

LEMMA 3.5. If the inequalities (3.8) and (3.9) are satisfied, then $\left(w_{0}, v_{0}\right)$ as given in (3.2) is an element of $H^{1}(\Omega) \times L^{2}(\Omega)$.

Proof. Clearly, (3.8) is equivalent to $v_{0} \in L^{2}(\Omega)$. (3.9) implies that $w_{0}$ given by (3.2) lies in the domain of the positive definite operator $(-\Delta)^{1 / 2}$ in $L^{2}(\Omega)$. Since $V^{\prime}$ has been identified with $L^{2}(\Omega)$, the operator $-\Delta$ defines an isomorphism of $V$ onto $L^{2}(\Omega)$, i.e. given $u \in V$ there is exactly one $w \in L^{2}(\Omega)$ such that $w=-\Delta u$ and vice versa. Moreover, there are constants $L_{0}, L_{1}>0$ such that

$$
L_{0}\|u\|_{H^{2}(\Omega)} \leq\|w\|_{L^{2}(\Omega)} \leq L_{1}\|u\|_{H^{2}(\Omega)} .
$$

The domain of $(-\Delta)^{1 / 2}$ is the interpolation space

$$
\left[V, L^{2}(\Omega)\right]_{1 / 2} \subset\left[H^{2}(\Omega), L^{2}(\Omega)\right]_{1 / 2}=H^{1}(\Omega) .
$$

Hence, (3.9) implies $w_{0}$ lies in the domain of $(-\Delta)^{1 / 2}$ which in turn is contained in $H^{1}(\Omega)$.

The proof is complete.

\section{Initial state observability for the wave equation}

In this section, we apply our main theorem to establish the initial state observability result for the wave equation subject to the mixed boundary condition.

Consider the initial boundary value problem, which consists of (2.5) with $\Omega$ and $\Gamma$ defined as before:

$$
\begin{gathered}
\partial w / \partial \nu(x, t)+\sigma w(x, t)=0, \quad x \in \Gamma, t \geq 0, \sigma>0 \text { fixed } \\
w(x, 0)=w_{0}(x) \in V, \quad \partial w / \partial t(x, 0)=v_{0}(x) \in L^{2}(\Omega)
\end{gathered}
$$

This problem is known [11, Chapter IV] to have a unique solution $w(x, t)$ with the properties

$$
w \in C\left([0, T] ; H^{1}(\Omega)\right), \quad \partial w / \partial t \in C\left([0, T], L^{2}(\Omega)\right) .
$$

The observing operator $H: H^{1}(\Omega) \times L^{2}(\Omega) \rightarrow H^{-1 / 2}(\Gamma)$ is defined by

$$
H(w(\cdot, t), \partial w / \partial t(\cdot, t))=\partial w / \partial t(\cdot, t) .
$$

Again, the trace theorem shows that $H$ is a bounded linear operator. The corresponding observation operator

$$
C: D(C) \subseteq H^{1}(\Omega) \times L^{2}(\Omega) \rightarrow L^{2}(\Gamma \times[0, T])
$$


is given by

$$
C\left(w_{0}, v_{0}\right)=H(w(\cdot, \cdot), \partial w / \partial t(\cdot, \cdot))=\partial w / \partial t(\cdot, \cdot) .
$$

Using regularity results (e.g. Theorem 2.5) for solutions of (2.5), (4.1), (4.2) together with the trace theorem it follows that $V \times H^{1}(\Omega) \subset D(C)$.

In order to study the observability problem $(2.5),(4.1),(4.2)$ via (4.3), we consider the dual control system:

$$
\frac{\partial^{2} z}{\partial t^{2}}-\sum_{K=1}^{n} \frac{\partial^{2} z}{\partial x_{K}^{2}}=0, \quad x \in \Omega, t \geq 0,
$$

with terminal conditions

$$
z(x, t)=\partial z / \partial t(x, T)=0
$$

and the boundary condition

$$
\partial z / \partial \nu(x, t)+\sigma z(x, t)=f(x, t), \quad x \in \Gamma, t \geq 0, \sigma>0 \text { fixed. }
$$

By using the divergence theorem together with the assumption that $w_{0} \in V$, it can be deduced from

$$
0=\int_{\Omega \times[0, T]}\left[\frac{\partial z}{\partial t}\left(\frac{\partial^{2} w}{\partial t^{2}}-\Delta_{n} w\right)+\frac{\partial w}{\partial t}\left(\frac{\partial^{2} z}{\partial t^{2}}-\Delta_{n} z\right)\right] d x d t
$$

that the following relationship holds:

$$
\begin{aligned}
-\int_{\Omega} & {\left[v_{0}(x) \frac{\partial z}{\partial t}(x, 0)+\sum_{K=1}^{n} \frac{\partial w_{0}(x)}{\partial x_{K}} \frac{\partial z(x, 0)}{\partial x_{K}}\right] d x } \\
= & \int_{\Gamma \times[0, T]}\left(\frac{\partial z}{\partial t}(x, t) \frac{\partial w}{\partial \nu}(x, t)+\frac{\partial z(x, t)}{\partial \nu} \frac{\partial w}{\partial t}(x, t)\right) d s d t \\
= & \int_{\Gamma \times[0, T]} \frac{\partial w}{\partial t}(x, t)(f(x, t)-\sigma z(x, t)) d s d t \\
& +\int_{\Gamma \times[0, T]} \sigma z(x, t) \frac{\partial w}{\partial t}(x, t) d s d t+\int_{\Gamma} \sigma z(x, 0) w(x, 0) d s
\end{aligned}
$$

Thus,

$$
\begin{aligned}
& \int_{\Gamma \times[0, T]} f(x, t) \frac{\partial w}{\partial t}(x, t) d s d t \\
&=-\int_{\Omega}\left(v_{0}(x) \frac{\partial z}{\partial t}(x, 0)+\sum_{K=1}^{n} \frac{\partial w_{0}(x)}{\partial x_{K}} \frac{\partial z(x, 0)}{\partial x_{K}}\right) d x \\
&-\int_{\Gamma} \sigma z(x, 0) w_{0}(x) d s .
\end{aligned}
$$

In particular, (4.7) holds when $(w(x, 0), \partial w / \partial t(x, 0))=\left(w_{0}, v_{0}\right) \in V \times H^{1}(\Omega)$ so that $\partial w / \partial t \in H^{1 / 2}(\Gamma \times[0, T]) \subset L^{2}(\Gamma \times[0, T])$, and for control functions 
$f \in L^{2}(\Gamma \times[0, T])$, which steers the state $(z(\cdot, 0), \partial z / \partial t(\cdot, 0)) \in H^{1}(\Omega) \times L^{2}(\Omega)$ to zero at time $T$.

Define $X \equiv\left\{(w, v):(w, v) \in H^{1}(\Omega) \times L^{2}(\Omega)\right\}$. Note that $X$ is a Hilbert space with the inner product

$$
\langle(w, v),(\tilde{w}, \tilde{v})\rangle_{X}=\int_{\Omega}\left(v(x) \tilde{v}(x)+\sum_{K=1}^{n} \frac{\partial w(x)}{\partial x_{K}} \frac{\partial \tilde{w}(x)}{\partial x_{K}}\right) d x+\int_{\Gamma} \sigma w(x) w(\tilde{x}) d s
$$

Let the associated norm be denoted by $\|\cdot\|_{X}$. Lemma 2.7 shows that this norm is equivalent to the usual norm in $H^{1}(\Omega) \times L^{2}(\Omega)$. Equation (4.7) shows that

$$
C: D(C) \subseteq H^{1}(\Omega) \times L^{2}(\Omega) \rightarrow L^{2}(\Gamma \times[0, T])=Y
$$

has an adjoint operator

$$
C^{*}: D\left(C^{*}\right) \subseteq L^{2}(\Gamma \times[0, T]) \rightarrow X^{*}=X=H^{1}(\Omega) \times L^{2}(\Omega),
$$

where

$$
C^{*} f=-(z(\cdot, 0), \partial z / \partial t(\cdot, 0))
$$

The right-hand side is the initial state to be steered to zero at time $T$ when the control $f$ is used in (4.4)-(4.6). The domain of $C^{*}$ consists of those $f$ for which

$$
(z(\cdot, 0), \partial z / \partial t(\cdot, 0)) \in X \text {. }
$$

From Theorem 2.4, we have the following controllability result for the system (4.4)-(4.6): If $T>2$, each initial state with

$$
\begin{aligned}
z(\cdot, 0) & =z_{0} \in H^{1}(\Omega), \\
\partial z / \partial t(\cdot, 0) & =z_{1} \in L^{2}(\Omega),
\end{aligned}
$$

(i.e. each initial state in $X$ ) can be steered to zero at the time $T$ by use of a control $f \in L^{2}(\Gamma \times[0, T])$. This means that $C^{*}$ maps from $D\left(C^{*}\right)$ onto $X$. Application of Theorem 2.3 part (b) with $X$ and $Y$ as indicated above yields

$$
\left\|\left(w_{0}, v_{0}\right)\right\|_{X} \leq K\left\|C\left(w_{0}, v_{0}\right)\right\|_{Y}
$$

for each $\left(w_{0}, v_{0}\right) \in D(C)$ and $T>2$. Thus the system given by (4.3) is observable.

\section{Observability and controllability of the heat equation}

In this section, we consider some observability problems for the heat equation. Our aim is to deduce the controllability result by using Theorem 2.3.

Let $\Omega$ and $\Gamma$ be defined as in Section 2. Consider the parabolic equation

$$
\frac{\partial w}{\partial t}-\sum_{K=1}^{n} \frac{\partial^{2} w}{\partial x_{K}^{2}}=0, \quad x \in \Omega, t \geq 0
$$


with the initial condition

$$
w(x, 0)=w_{0}(x) \in L^{2}(\Omega)
$$

and the boundary condition

$$
\partial w / \partial \nu(x, t)+\sigma w(x, t)=0, \quad x \in \Gamma, t \geq 0, \sigma>0 \text { fixed. }
$$

It is well hrouwn (see [13, Chapter VI, $\hat{\jmath} 2$, Theorems $\mathbf{i}$ and 3$\}$ ) that the unique solution $w$ of $(5.1)-(5.3)$ is an element in $L^{2}\left(0, \tau ; H^{1}(\Omega)\right)$. Introducing the observing operator

$$
H: H^{1}(\Omega) \rightarrow L^{2}(\Gamma), \quad H w(\cdot, t)=\left.w(\cdot, t)\right|_{x \in \Gamma}
$$

the observation operator $C$ is defined by

$$
C: w_{0} \in L^{2}(\Omega) \rightarrow w \in L^{2}(\Gamma \times[0, \tau])
$$

for some fixed $\tau>0$.

In conjunction with (5.1)-(5.3), we consider the controlled parabolic process:

$$
\begin{aligned}
\partial z / \partial t-\Delta_{n} z & =0, \quad x \in \Omega, t \geq 0 \\
z(x, 0) & =0 \\
\partial z / \partial \nu(x, t)+\sigma z(x, t) & =f(x, t), \quad x \in \Gamma, t \geq 0, \sigma>0 \text { fixed, }
\end{aligned}
$$

where $f \in L^{2}(\Gamma \times[0, \tau])$. Using the divergence theorem, we obtain

$$
\begin{aligned}
0 & =\int_{\Omega \times[0, T]} w(x, \tau-t)\left(\partial z / \partial t-\Delta_{n} z\right) d x d t \\
& =\int_{\Omega} w_{0}(x) z(x, \tau) d x-\int_{\Gamma \times[0, \tau]} w(x, \tau-t) f(x, t) d s d t .
\end{aligned}
$$

Substituting $u=\tau-t$ and replacing $u$ by $t$, we get

$$
\begin{aligned}
\int_{\Omega} w_{0}(x) z(x, \tau) d x & =\int_{\Gamma \times[0, \tau]} w(x, t) f(x, \tau-t) d s d t \\
& \equiv \int_{\Gamma \times[0, \tau]} w(x, t) h(x, t) d s d t .
\end{aligned}
$$

Since $w \in L^{2}\left([0, \tau] ; H^{1}(\Omega)\right)$ it follows from the trace theorem that

$$
\left.w\right|_{\Gamma} \in L^{2}(\Gamma \times[0, \tau]) \text {. }
$$

Let $X \equiv L^{2}(\Omega), Y \equiv L^{2}(\Gamma \times[0, \tau])$, and defining $C$ by $(5.4)$, the dual operator $C^{*}$ is

$$
C^{*}: h(x, t)=f(x, \tau-t) \in L^{2}(\Gamma \times[0, \tau]) \rightarrow z(\cdot, \tau) \in L^{2}(\Omega) .
$$

This means that $C^{*}$ takes $h(x, t)$ into the final state $z(\cdot, \tau)$ when the control $f$ is used in (5.5)-(5.7). 
Let $-\lambda_{K}<0, K=1,2, \ldots$, denote the eigenvalues of the Laplace operator subject to the mixed boundary condition. Let the orthonormalized eigenfunctions in $L^{2}(\Omega)$ be denoted by $\phi_{K, l}, K=1,2, \ldots, l=1,2, \ldots, m_{K}$, where $m_{K}$ is the multiplicity of the eigenvalue $-\lambda_{K}$. Then,

$$
C \phi_{K, l}=\left.e^{-\lambda_{K} t} \phi_{K, l}\right|_{\Gamma} .
$$

In [15], it is shown that the solution $g_{K, l}(x, t)$ of the moment problem for the parabolic control problem (5.5)-(5.7) can be constructed from the solution $\hat{g}_{K, l}(x, t)$ of the corresponding moment problem for the hyperbolic control problem (4.4)-(4.6) by Fourier transformation. There it is shown that the functions $g_{K, l}(x, t), x \in \Gamma, K=1,2, \ldots, l=1, \ldots, m_{K}$ form a biorthogonal set with respect to the functions

$$
\phi_{K, l}(x) \exp \left(-\lambda_{K} t\right) \quad \text { in } L^{2}(\Gamma \times[0, \tau]),
$$

that is

$$
\delta_{K, l}^{i, j}=\int_{\Gamma \times[0, \tau]} \phi_{i, j}(x) \exp \left(-\lambda_{K} t\right) g_{K, l}(x, t) d s d t .
$$

Recall inequality (6.17) of [15]:

$$
\left\|g_{K, l}\right\|_{L^{2}(\Gamma \times[0, \tau])}^{2} \leq C(\tau) \mu(\Gamma) \exp \left(M \lambda_{K}^{1 / 2}\right),
$$

where $C(\tau)$ is a constant depending on $\tau, M=$ const. $>0, \mu=$ Lebesgue measure. It follows from (5.8) together with the Cauchy-Schwarz inequality that

$$
1 \leq\left\|g_{K, l}\right\|_{L^{2}(\Gamma \times[0, \tau])} \cdot\left\|\phi_{K, l}(x) \exp \left(-\lambda_{K} t\right)\right\|_{L^{2}(\Gamma \times[0, \tau])} .
$$

Hence, using inequality (5.9), we obtain for each $K=1,2, \ldots, l=1,2, \ldots, m_{K}$,

$$
K(\tau)\left\|\phi_{K, l}(x) \exp \left(-\lambda_{K} t\right)\right\|_{L^{2}(\Gamma \times[0, \tau])} \geq\left\|\exp \left(-M \lambda_{K}^{1 / 2}\right) \phi_{K, l}(x)\right\|_{L^{2}(\Omega)},
$$

since $\phi_{K, l}$ is chosen to be orthonormal in $L^{2}(\Omega)$ and $K(\tau)$ is a constant depending only on $\tau$.

From inequality (5.10), we can easily establish the observability results. More precisely, inequality (5.10) means that if we define $F: X \rightarrow X$ by

$$
F=\exp \left[-M(-\Delta)^{1 / 2}\right]
$$

then we have $F$-observability, that is

$$
K\left\|C w_{0}\right\|_{Y} \geq\left\|F w_{0}\right\|_{X}, \quad w_{0} \in D(C) \subseteq X .
$$

Note that $(-\Delta)^{1 / 2}$ is well defined, since $-\Delta$ is a positive-definite self-adjoint operator. Replacing $F$ by $\tilde{F} \equiv \exp (\tau \Delta)$, final state observability for the system (5.1)-(5.3) holds if $K$ is replaced by some $\tilde{K}>0$. This is due to the fact that $\exp \left[-M(-\Delta)^{1 / 2}\right]$ and $\exp (\tau \Delta)$ are positive-definite self-adjoint operators with eigenvalues $\exp \left(-M \lambda_{K}^{1 / 2}\right)$ and $\exp \left(-\tau \lambda_{K}\right)$ respectively, where

$$
\lim _{K \rightarrow \infty} \lambda_{K} \rightarrow \infty \text {. }
$$


Thus, this final state observability result agrees with those obtained in [6], [7], [14], [19] and [21].

From Theorem 2.3, part (a), we see that the range of the operator $C^{*}$ includes the range of $F^{*}$ (or of $\tilde{F}^{*}$ ), that is

$$
R\left(\exp \left[-M(-\Delta)^{1 / 2}\right]\right)=R\left(F^{*}\right) \subseteq R\left(C^{*}\right) .
$$

This inclusion means that the set of final states $z(\cdot, \tau)$, which may be reached from $z(\cdot, 0)=0$ by means of a control $f \in L^{2}(\Gamma \times[0, \tau])$, includes states $\Lambda$ defined by:

$$
\Lambda=\left\{z(\cdot, \tau): \sum_{K=1}^{\infty} \sum_{l=1}^{m_{k}} \alpha_{K, l} \phi_{K, l}\right\}
$$

where

$$
\sum_{k=1}^{\infty} \sum_{l=1}^{m_{k}}\left|\alpha_{K, l}\right|^{2}\left\|\phi_{K, l}\right\|_{L^{2}(\Gamma \times[0, \tau])}^{2}<\infty .
$$

In view of the estimate (5.9), (5.12) can be replaced by

$$
\sum_{k=1}^{\infty} \sum_{l=1}^{m_{k}}\left|\alpha_{K, l}\right|^{2} \exp \left[2 M \lambda_{K}^{1 / 2}\right]<\infty .
$$

An interpretation of the condition (5.13) can be found in [6]. Furthermore, it follows from Corollary 3.2 and Theorem 3.3 of [6] that this condition is, in a certain sense, necessary and sufficient for the validity of (5.11).

\section{Acknowledgement}

The author wishes to thank Prof. J. Wloka for providing a sketch of the proof that $V$ is dense in $H^{1}(\Omega)$. This paper was written during the author's stay at the School of Mathematics, University of New South Wales, N.S.W. 2033, Australia.

\section{References}

[1] N. U. Ahmed and K. L. Teo, Optimal control of distributed parameter systems (North Holland, 1981).

[2] S. Dolecki, "Observability for the one-dimensional heat equation", Studia Math. 48 (1973), 291-305.

[3] S. Dolecki, "Observability for Regular Processes", J. Math. Anal. Appl. 58 (1977), 178188.

[4] S. Dolecki and David L. Russell, "A general theory of observation and control", SIAM J. Control. Optim. 15 (1977), 185-220.

[5] A. Erdélyi, W. Magnus, F. Oberbettinger and F. G. Tricomi, Higher Transcendental Functions, Vol. II (McGraw-Hill, New York, 1953). 
[6] H. O. Fattorini and David L. Russell, "Exact controllability theorems for linear parabolic equations in one space dimension", Arch. Rational Mech. Anal. 4 (1971), 272-292.

[7] H. O. Fattorini and David L. Russell, "Uniform bounds on biorthogonal functions for real exponentials with an application to control theory of parabolic equations", Quart. Appl. Math. 32 (1974), 45-69.

[8] K. D. Graham, "Separation of eigenvalues of the wave equation for the unit ball in $\mathbf{R}^{N \text { ", }}$ Stud. Appl. Math. 52 (1973), 329-344.

[9] K. D. Graham and David L. Russell, "Boundary value control of the wave equation in a spherical region", SIAM J. Control. Optim. 13 (1975), 174-196.

[10] John Lagnese, "Control of wave processes with distribution controls supported on a subregion", SIAM J. Control. Optim. 21 (1983), 68-85.

[11] J. L. Lions, Optimal control of systems governed by partial differential equations (Springer Verlag, New York, 1971).

[12] N. Levinson, "Gap and density theorems", Colloguium Publications 26, Amer. Math. Soc. (1940).

[13] V. P. Mikhailov, Partial Differential Equations (English Translation) (Mir Publishers, Moscow, 1978).

[14] V. J. Mizel and T. J. Seidman, "Observation and prediction for the heat equation II", $J$. Math. Anal. Appl. 38 (1972), 149-166.

[15] David L. Russell, "A unified boundary controllability theory for hyperbolic and parabolic differential equations", Stud. Appl. Math. 52 (1973), 189-211.

[16] David L. Russell, "Exact boundary value controllability theorems for wave and heat processes in star-complemented regions", Proc. Conf. of Differential Games and Control Theory, 291-319, Kingston, R. J. (Marcel Dekker, New York, 1974).

[17] David L. Russell, "Nonharmonic Fourier series in the control theory of distributed parameter systems", J. Math. Anal. Appl. 18 (1967), 542-560.

[18] David L. Russell, "Control theory of hyperbolic equations related to certain questions in harmonic analysis and spectral theory", J. Math. Anal. Appl. 40 (1972), 336-368.

[19] T. J. Seidman, "Observation and Prediction for One-Dimensional Diffusion Equations", J. Math. Anal. Appl. 51 (1975), 165-175.

[20] H. Triebel, Höhere Analysis (Verlag Harri Deutsch, Frankfurt am Main, 2. Auflage, 1980).

[21] K. D. Werner, "An observation problem for the Bessel differential operator", J. Austral. Math. Soc. Ser. B 26 (1984), 92-107.

[22] K. D. Werner, "Boundary value control problems involving the Bessel differential operator", J. Austral. Math. Soc. Ser. B 27 (1986), 453-471.

[23] J. Wloka, Partielle Differentialgleichungen (B. G. Teubner, Stuttgart, 1982). 